\title{
Distributed Energy Resources (DER) Systems Integration into MV and LV Networks - Impact on Power Quality of the System
}

\author{
J. Stenzel ${ }^{1}$ and E. Vilchez ${ }^{2}$ \\ ${ }^{1}$ Head of Department of Power System Control \\ Institute of Electrical Energy Systems, Technische Universität Darmstadt \\ Landgraf-Georg-Str. 4, 64283 Darmstadt (Germany) \\ Phone/Fax number: +49(0)6151162852/4259, e-mail: juergen.stenzel@eev.tu-darmstadt.de \\ ${ }^{2}$ Department of Power System Control \\ Institute of Electrical Energy Systems, Technische Universität Darmstadt \\ Landgraf-Georg-Str. 4, 64283 Darmstadt (Germany) \\ Phone/Fax number: +49(0)6151165151/4259, e-mail: elda.vilchez@eev.tu-darmstadt.de
}

\begin{abstract}
The fast growing of Distributed Generation (DG) in European electricity networks, lead to challenges of different nature. Due the fast growth of market split of Distributed Energy Resources (DER), today's electric power system of centrally located generation, transmission networks and distribution networks is expected to evolve into a new configuration. The achievement of such drastic transformation will heavily rely on studies that evaluate the behaviour of networks with integrated DER systems. In particular, Power Quality (PQ) has been given significative attention due to the fact that new power system applications are more and more sensitive to power quality disturbances.
\end{abstract}

The aim of this study is to examine the power quality behaviour of the network in case of integration of DER units at different locations of the MV and LV system. In order to analyse the impact of DG on the network's power quality, a $380 \mathrm{kV}$ transmission system with MV, and LV subsystems, $10 \mathrm{kV}$ and $0.4 \mathrm{kV}$ respectively is used, DER units are integrated and power quality analyses are performed.

\section{Key words}

Distributed generation, $380 \mathrm{kV}$ transmission system with MV and LV subsystems, distributed energy resource units, power quality analysis.

\section{Introduction}

The growing integration of DG leads to fundamental changes in the power generation systems. The conventional central structures are now being converted to a distributed system where unidirectional power flows are now reversed under the influence of small DER units feeding in at medium and low voltage level of the network. These DER systems also involve sensitive device, power electronic interfaces, as well as communications and control devices. Therefore, the integration of DER units will create new issues regarding power quality. In this context, the impact of these power sources on the network's power quality is analyzed. Based on The IEEE P1547, a norm explaining the technical specifications and requirements to interconnect generators with aggregate capacity of 10 MVA or less with the electric power system, the DER units are integrated in the system [1].

The goal of this study is to examine the effect of the integration of DER units at the Medium Voltage (MV) and Low Voltage (LV) system and to analyze the impact on the power quality on the network. In this framework a model of a $110 \mathrm{kV}$ transmission system and DER has been modelled and analyzed with the help of a commercial power system planning simulation software [2] and the standard EN 50610 [3]. This research shows the possibilities to maintain or even improve the power quality in networks with distributed generation.

\section{Network Modelling}

The basic model contains a $380 \mathrm{kV}$ transmission system, in-feed at different points with conventional power plants and is connected with a $380 \mathrm{kV}$ external network. Additionally the $380 \mathrm{kV}$ network feeds a subtransmission systems with $110 \mathrm{kV}$ transmission lines, 10 $\mathrm{kV}$ and $0.4 \mathrm{kV}$ distribution systems, where the DER units have been integrated.

In order to present a general overview of the network a block design of the whole power system model has been created (Fig 1). 


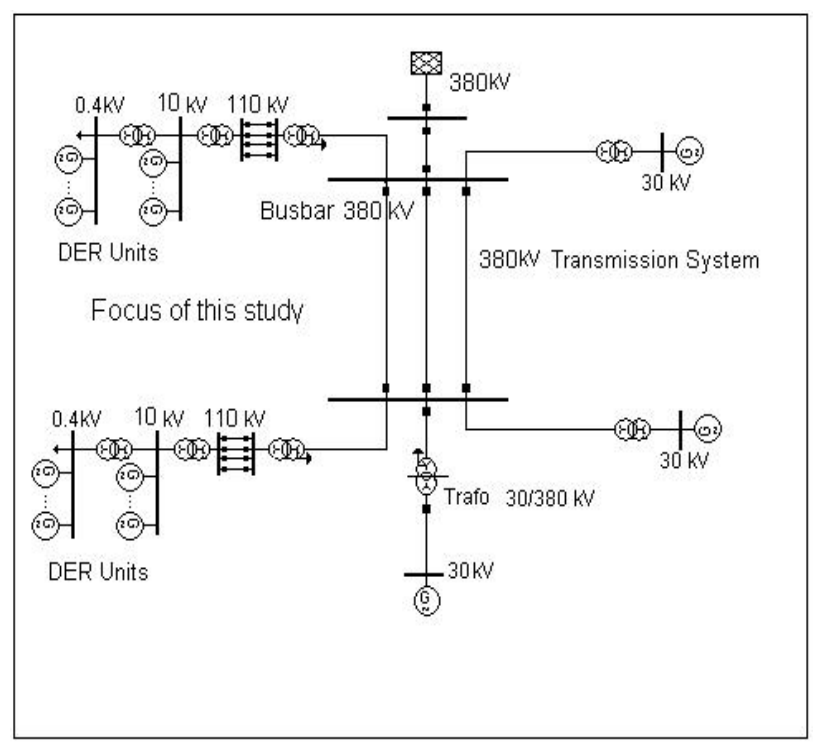

Fig. 1. Block design of the power system model.

This test network has been used for different studies in power quality [4], [5]. It has been extended for this study by integrating DER units. The block design contains the following significant parts that compose the network: the $380 \mathrm{kV}$ transmission system, the MV and LV systems and the DER units' models. These blocks will be explained subsequently.

\section{A. The 380 kV Transmission System}

The $380 \mathrm{kV}$ transmission system is a balanced network with $850-\mathrm{km}$ three-phase overhead lines, a connection to one external grid and four different power plants, connected to $380 \mathrm{kV}$ substations. The four conventional power plants together have a total installed capacity of $800 \mathrm{MW}$. The subsystems, $110 \mathrm{kV}, 10 \mathrm{kV}$ and $0.4 \mathrm{kV}$, are fed from the network with the conventional synchronous generators and distributed generation. (Fig. 2).

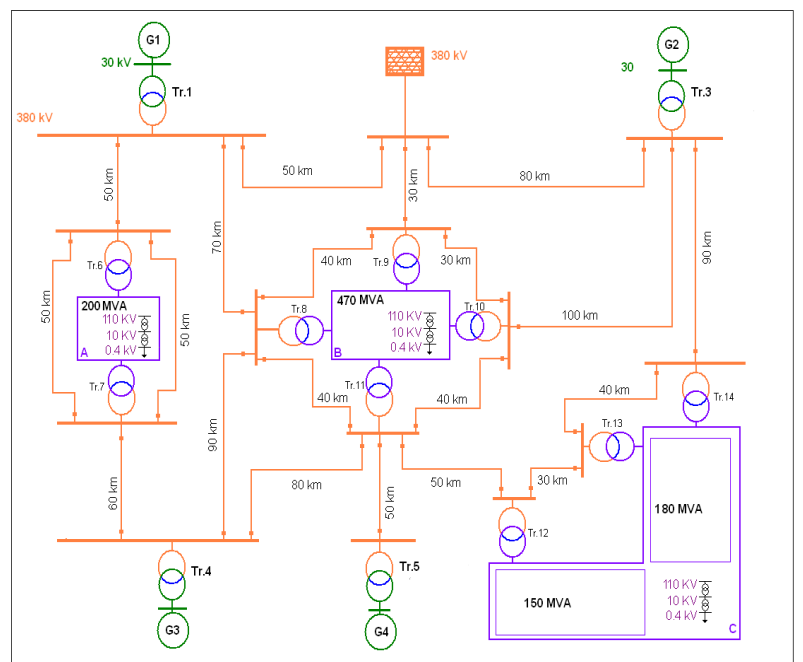

Fig. 2. Block design of the power system model.

Three load blocks, which represent the load centres, have been designed. The total load of the system is about 1000 MVA. Each load block has a different configuration.

\section{B. The MV and LV System}

The subsystems, $10 \mathrm{kV}$ and $0.4 \mathrm{kV}$, are fed from the $110 \mathrm{kV}$ transmission grid which is directly connected with the $380 \mathrm{kV}$ system.

The total load of the three load blocks system is 1000 MVA active power. Each load block has a different construction in order to test different network configurations. To keep the computational effort as low as possible and taking into account some software limitation, each load block is analyzed separately while the other two blocks are represented by equivalent loads. For this study only load block A is considered.

Load block A, has a central fed system with two branch connected with the $380 \mathrm{kV}$ level and a total consumption of 200 MVA active power. The two branches are almost identical with two step-down transformers connecting the $110 \mathrm{kV}$ subsystem with the $380 \mathrm{kV}$ level. The MV and LV systems served by step-down transformers are represented by one busbar each with concentrated load. The difference between the two branches is that one branch is connected on one size of the system with the 10 $\mathrm{kV}$ and $0.4 \mathrm{kV}$ levels to the loads and the DER system while on the order size only to the loads. This case will be interesting in order to compare the behaviour of the system on both sides, with and without DER units.

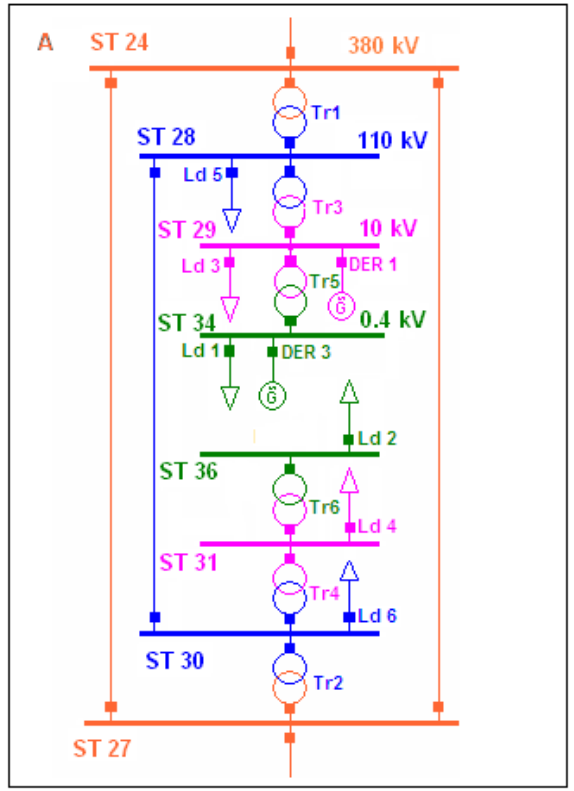

Fig. 3. Load Block A.

\section{Distributed energy resource (DER) systems}

Distributed energy resource (DER) systems are smallscale power generation technologies (typically in the range of $3 \mathrm{~kW}$ to $10,000 \mathrm{~kW}$ ) used to provide an alternative to or an enhancement of the traditional electric power system [1]. DER systems include microturbines, CHP, fuel cells, solar panels, bio-energy, and small wind energy systems integrated in the system (Fig.4). 


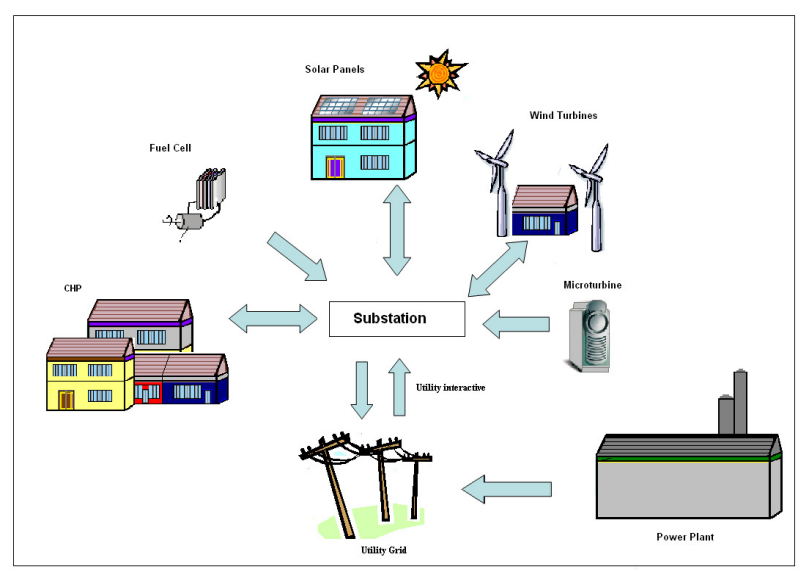

Fig. 4. DER System.

As the focus of this study is only on load block A, just 2 types of DER systems are contemplated.

1) Wind Turbine Model. It was assumed for the model that the wind mills have a rated power of about $5 \mathrm{MVA}$ and is interconnected to the $10 \mathrm{kV}$ system. The wind turbine used in this research is based on the doubly-fed induction generator concepts (DFIG, Fig.4). The main components of the DFIG are: induction generator model, PWM converters and pitch control. Furthermore, it is assumed that the wind-farms are equipped with low-voltage ride-through capability and reactive current support according to the connection standards [5].

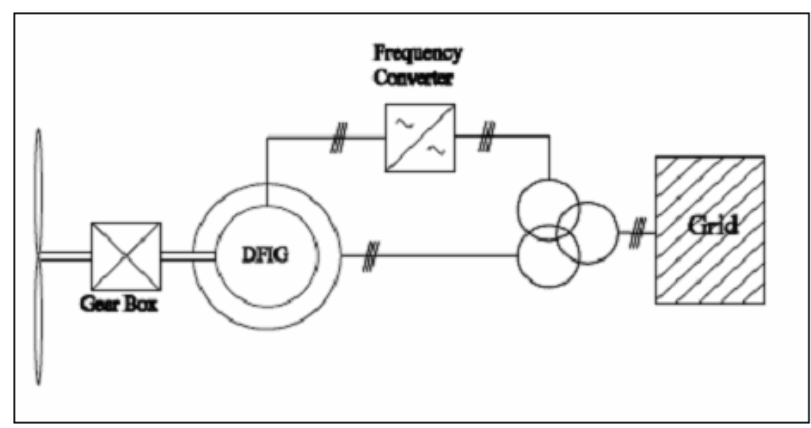

Fig. 4. Doubly-Fed-Induction-Generator

2) Microturbine Model. It was assumed for the model that microturbines have a rated power of about $55 \mathrm{~kW}$ and are interconnected to the 0.4 $k V$ system. The microturbines used in this research are of a high-speed single-shaft design with the compressor and turbine mounted on the same shaft as the permanent magnet synchronous generator. The generator generates a very high frequency three phase signal ranging from 1500 to $4000 \mathrm{~Hz}$. The high frequency voltage is first rectified and then inverted to a normal 50 or $60 \mathrm{~Hz}$ voltage

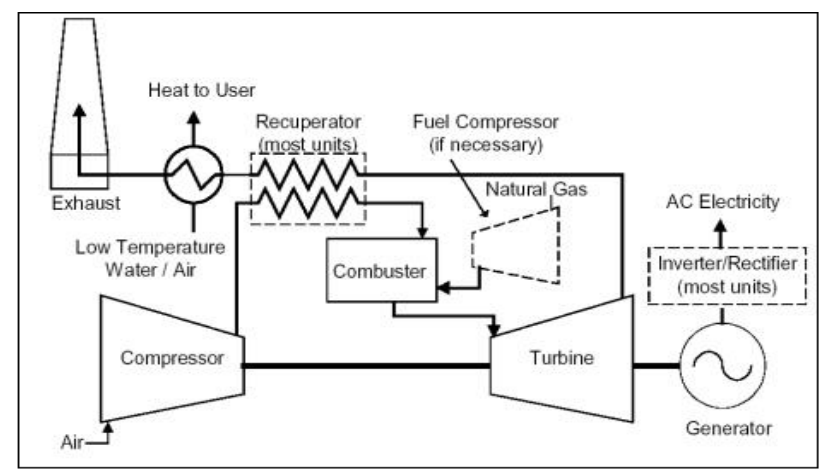

Fig. 5. Microturbine generation system.

\section{Power Quality Analysis}

For the power quality analysis different scenarios have been designed. In previous studies the behaviour of the system in case of wind integration was analyzed [4]. As it has been shown, the integration of wind energy has a considerable impact on the power quality of the system.

A base case is created to show the behaviour of the system with four conventional power plants and the DER units. The base case has been modified and several cases, where the four conventional power plants are changed with wind farm with different amount of wind energy had been created [4].

The scenarios investigated are:

"Base Case": All generators are conventional synchronous generators directly connected to the transmission system. Load block A is analyzed (Fig. 7). This case will be interesting in order to observe the behaviour of the load block A system in both sides, with DER units, Brach1 and without DER units Branch2.

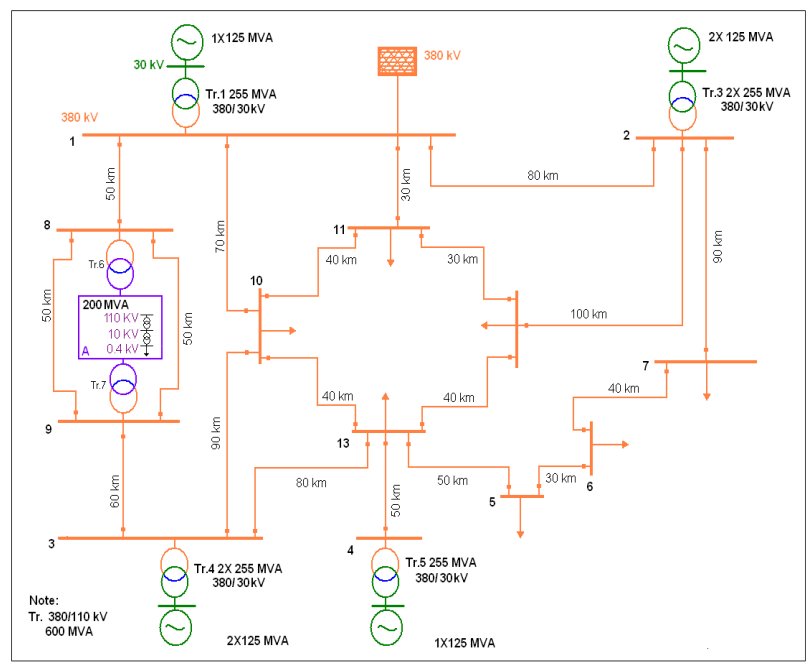

Fig. 7. Base Case Scenario

The base case has been modified. Several cases, where the four conventional power plants are replaced with wind farm have been created and the amount of wind energy integration is changed: case 1:100\% WE, case 2: 75\% WE, case 3: 50\% WE and case 4: 30\% WE. 


\section{A. Voltage Sags Analysis}

The voltage sags analysis uses fault statistics to describe the frequency of errors and uses these statistics to weight the result of each event and calculate the overall effect of failures. Through this foundation sags assessment tables for each scenario were calculated, the results showed the low penetration of voltage sags and these results were similar for the three scenarios and the different cases.

This was expected as the low-voltage ride-through capability reduces the effects of voltage sags on the network. Therefore these results were not further discussed [4].

\section{B. Harmonic Analysis}

A harmonic analysis is performed using the simulation software [2]. A harmonic penetration was assessed in the $380 \mathrm{kV}$ transmission networks. The HD \% on the MV $(10 \mathrm{kV})$ and $\mathrm{LV}(0.4 \mathrm{kV})$ is analysed at this point.

The European standard EN 50160 [3] specifies $8 \%$ as the limit for the total harmonic voltage distortion (THD \%) on the supply voltage. The THD \% was measured on the MV and LV substations ( $\mathrm{St}$ ) for both side of the system. The subsequent results on the system with DER units, Branch1, St29 and St34; and the other side without DER system Branch2, St31 and St36 station are shown in figure 8 below.

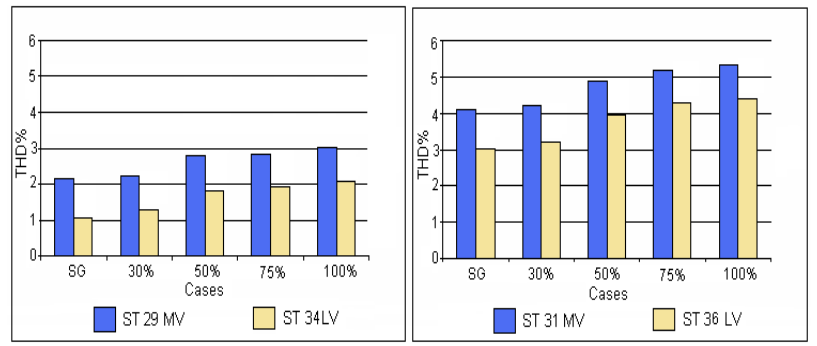

Fig. 8. Total harmonic distortion "Branch 1" and "Branch 2"

Two tendencies can be noted; first in "Branch1" the maximum percentage of THD is $3.18 \%$ and $2.17 \%$ on the St 29 and St 34 respectively. These two maximum values are present when $100 \% \mathrm{WE}$ is integrated in the system. The THD is always bigger in St 29 where the DER 1 Unit (small wind system) is integrated and smaller on St 34 where the DER 3 unit (Microturbine system) is integrated. This is of interest as the results show an increase of the THD\% when more wind energy is integrated. Related with "Branch 2", a similar trend to the trend in "Scenario1_WFA" is detected but with the difference that on the $100 \%$ case. The maximum percentage of the THD is $5.44 \%$ and $4.57 \%$ on the St 29 and St 36 respectively. These results show that "branch 1 " has less total harmonic distortion. Based on this it is assumed that this branch has better accuracy than branch 2. It is proven that the branch with DER units' integration has a better response for the quality perturbation. It is also proven that inclusive with $100 \%$ wind energy integration the system is working within the allowed limits since the maximum THD percentage stays below the EN 50160 limit of $8 \%$

In order to examine the behavior of the system in both branches of the "Load Block A" the HD \% on the St 29 and St 31 (MV 10kV) and St 34 and St $36(\mathrm{LV} 0.4 \mathrm{kV})$ were analyzed.

\section{"Branch 1":}

Figure 9 shows the results of the MV and LV system:
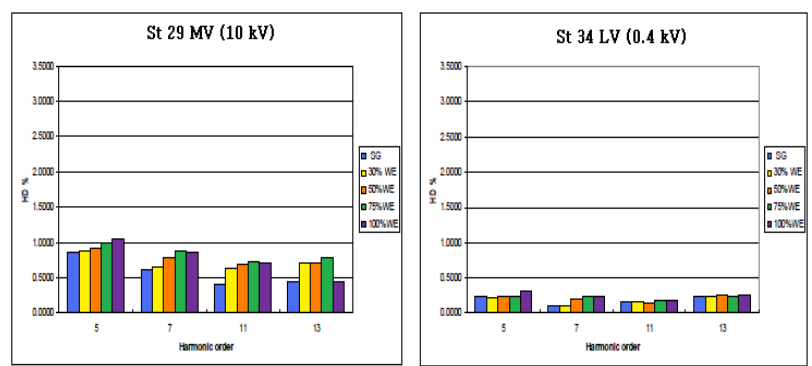

Fig. 9. Harmonic distortion on "Branch 1"

The dominating distortion has been identified as the 5th harmonics. The HD percentage is different in the different cases but it's appreciated that in St29 where the DER1 unit (small wind system) is integrated has a notable larger amount of HD than the St 34 where the DER 3 unit (Microturbine system) is integrated. This is of interest as the results show a decrease of the HD when Microturbines are integrated in the system. The results of the harmonic distortion are within the boundaries of the IEEE 519-1992 [8].

\section{"Branch 2":}

The results in branch 2 show how the system without DER units has a bigger amount of HD\% than the system with DER units. Based on this it is assumed that branch 1 has better responses against HD than branch 2 .
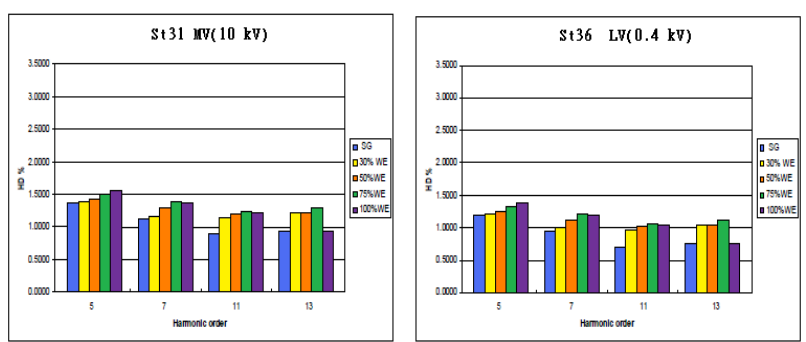

Fig. 10. Harmonic distortion on "Branch 2"

\section{Conclusion}

Based on this study, the impacts of the DER system integration on power quality have been studied and the influence on the grid's power quality of DER units' integration was measured on the low- and medium voltage level.

Power quality analyses have been performed at the transmission and distribution systems as well. Power quality measurements such as voltages sags and 
harmonics have been analyzed. The behaviour at the different levels of the system has been studied.

Although it is well-known that DFIG have a better influence on the power quality than other wind turbine generators and that DFIG characteristics introduce some improvements on the harmonics index results, it can be seen that the harmonic content influences on the output power terms. This is demonstrated with the increase of THD\% and HD\% on the station with small wind system integrated on the system.

CHP system operating continuously provides a greater level of protection for the customer against external voltage sags and other momentary disruptions. The CHP system is essentially a second feed for the customer.

The results up to this point demonstrate that the influence of increasing DG reinforced the system and provides a greater level of protection for the user side against power quality perturbations.

All procedures are being carried out with a model. Therefore it is necessary to take into account the limitations of the simulations and it will be interesting for future developments to compare the results with real case studies in order to prove the accuracy of the simulations. The analysis made will be used for future research as reference for different developments and solutions in the DG integration on networks with different voltage levels.

\section{References}

[1] IEEE P1547. Standard for Interconnecting Distributed Resources with Electric power system.

[2] DIgSILENT Power Factory Version 13.1.257, Educational Versions.

[3 ]EN 50160 Voltage characteristics of electricity supplied by public distribution systems, German version, DIN EN standard 50160:199.

[4] E. Vilchez, J. Stenzel. "Wind energy integration into $380 \mathrm{kV}$ system - Impact on power quality of MV and LV networks" in proc. 2008 International Conference on Renewable Energy and Power Quality (ICREPQ’08), Paper No. 350. Santander, Spain.

[5] E. Vilchez, J. Stenzel. "Comparison of wind energy integration into a $110 \mathrm{kV}$ and a $380 \mathrm{kV}$ transmission system Impact on power quality of MV and LV networks" in proc. 2009. IEEE PES Transmission and Distribution Conference and Exposition, Paper No.350. Wuhan, China.

[6] Spain K. Strunz. Developing Benchmark Models for Studying the Integration of Distributed Energy Resources. Power Engineering Society General Meeting, June 2006.

[7] E.ON - Netz. IGrid Code - High and Extra High Voltage". August 2003. www.eon-netz.com

[8] IEEE 519-1992 Recommended Practices and Requirements for Harmonic Control in Electrical Power Systems.

\section{Bibliography}

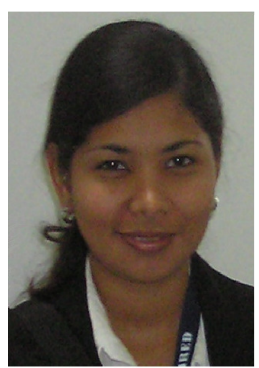

Elda Vilchez (1979) received Master Degree in Electrical Power Engineering, from the Darmstadt University of Technology, Germany, in 2006. She is currently doing her $\mathrm{PhD}$ thesis at Darmstadt University of Technology, Department of Electrical Engineering and Information Technology, Electrical Power System Laboratory in the area of power quality and renewable energy. Her research interests are power quality, distributed generation and wind integration.

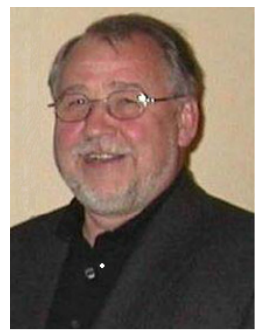

Juergen Stenzel (1944) received Dipl.Ing. degree in Electrical Engineering, from Munich University of Technology, Germany, in 1970, and the Dr.-Ing. Degree from the University of Siegen, Germany, in 1984. Currently, he is in the Darmstadt University of Technology, Germany, where he received a full professorship in the Department of Electrical Engineering and Information Technology. He is a member of the Electrical Power System Laboratory. His area of interest is power system analysis and control. 